\title{
RGB arrays for micro-LED applications using nanoporous GaN embedded with quantum dots
}

Jin-Ho Kang, ${ }^{1 \# *}$ Bingjun Li, ${ }^{1 \#}$ Tianshuo Zhao, ${ }^{2,3}$ Muhammad Ali Johar, ${ }^{4}$ Chien-Chung Lin, ${ }^{5}$ Yen-Hsiang Fang, ${ }^{5}$ Wei-Hung Kuo, ${ }^{5}$ Kai-Ling Liang, ${ }^{5}$ Shu Hu, ${ }^{2,3}$ Sang-Wan Ryu, ${ }^{4}$ and Jung Han ${ }^{1}$

${ }^{1}$ Department of Electrical Engineering, Yale University, New Haven 06520, United States

${ }^{2}$ Department of Chemical and Environmental Engineering, Yale University, New Haven 06520, United States

${ }^{3}$ Energy Sciences Institute, Yale University, West Haven 06516, United States

${ }^{4}$ Department of Physics, Chonnam National University, Gwangju 61186, Republic of Korea

${ }^{5}$ Electronic and Optoelectronic System Research Laboratories, Industrial Technology Research Institute ITRI, Hsinchu, Taiwan

(a)

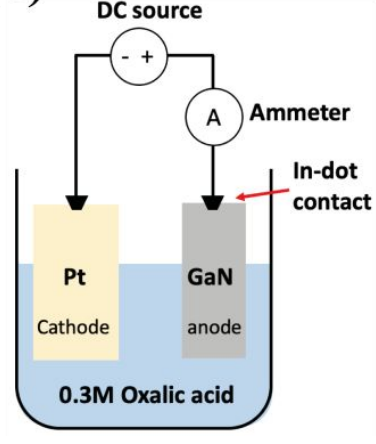

(b)

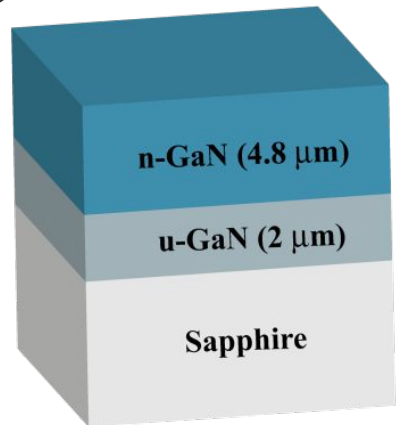

(c)

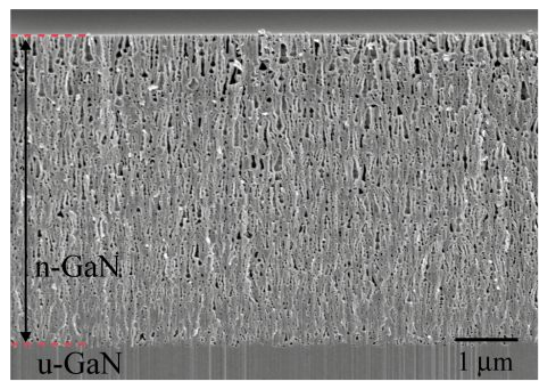

Figure s1. (a) Schematic images of EC etching setup and (b) GaN layers grown on sapphire and (c) cross-sectional SEM image of NP GaN. 
(a)

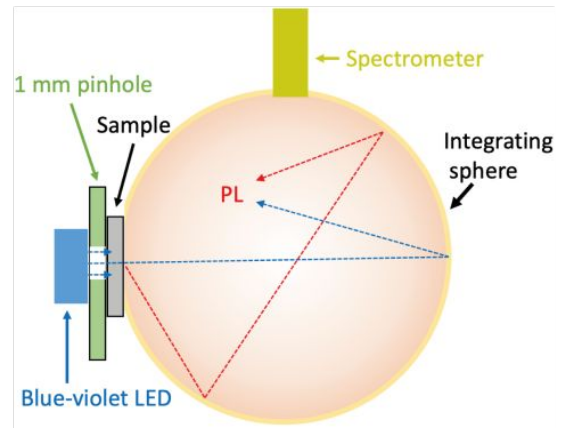

(b)

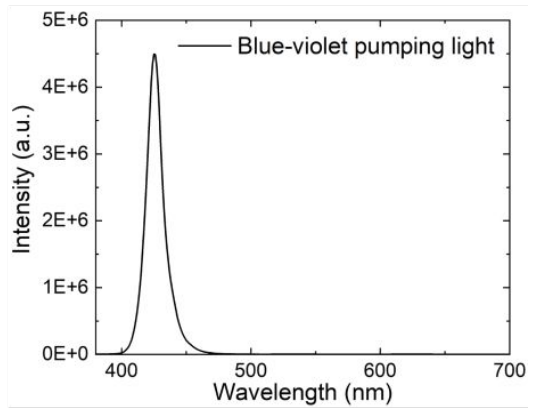

Figure s2. (a) Schematic diagram of LCE measurement setup and (b) luminescent spectrum of the blue-violet LED used to excite QD films and NPQDS.

(a)

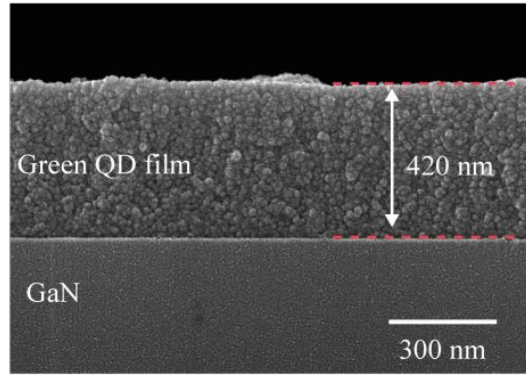

Figure S3. SEM images of dried (a) green and pristine GaN templates and (c) their effective qua defined as the ratio between blue-violet photons absorbed and green (or red) photons generated.

(c)

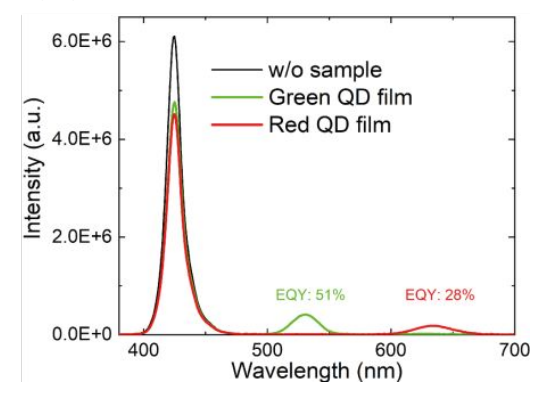
(b) red QD films on (b)

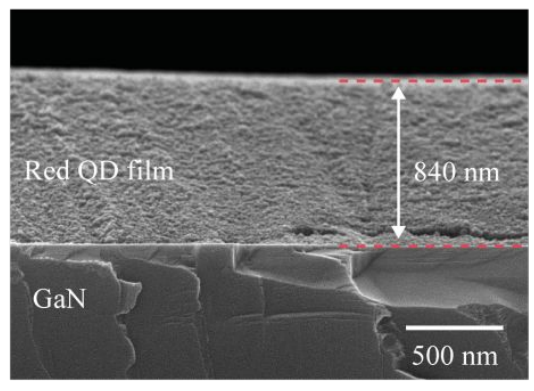
d d 
Figure S4. SEM images of NP GaN embedded with silica nanoparticles.

(a)

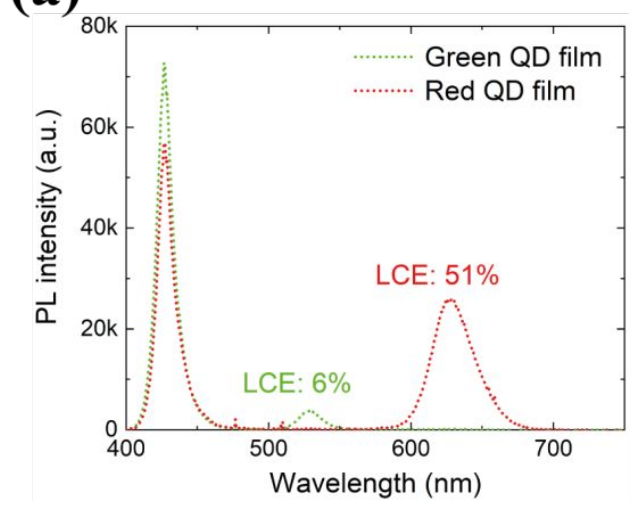

(b)

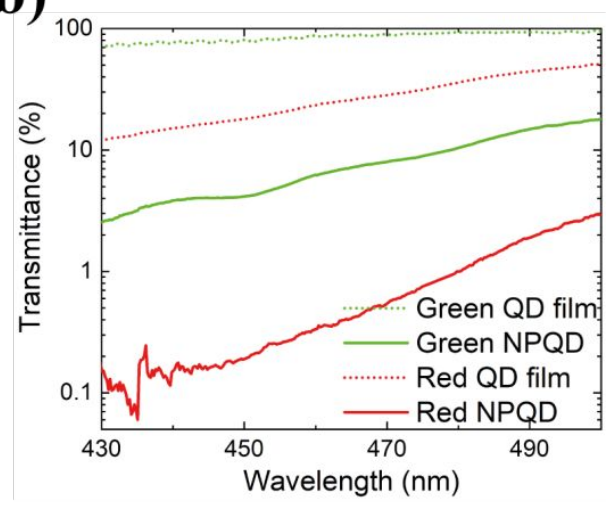

Figure S5. (a) Photoluminescent spectra of 420-nm-thick green and 840nm-thick red $Q D$ films. (b) Total transmittance spectra of (dotted) $Q D$ films and (solid) NPQDs.

(a)

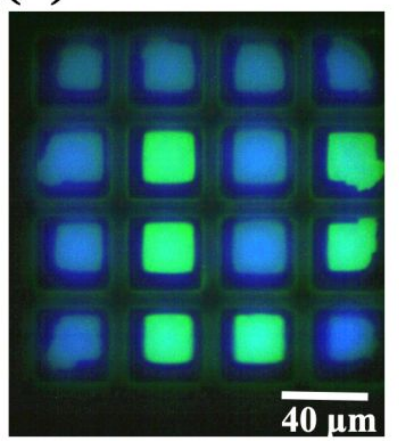

(b)

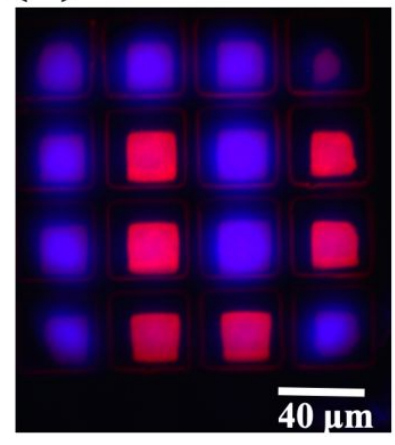

(c)

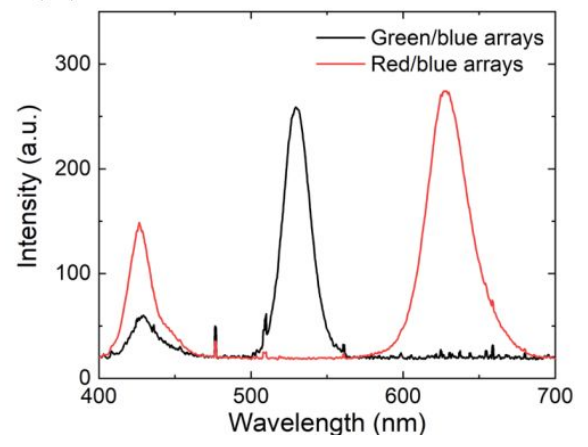

Figure s6. Optical microscope images of (a) green/blue and (b) red/blue arrays under the blue-violet pumping LED and (c) their PL spectra. 


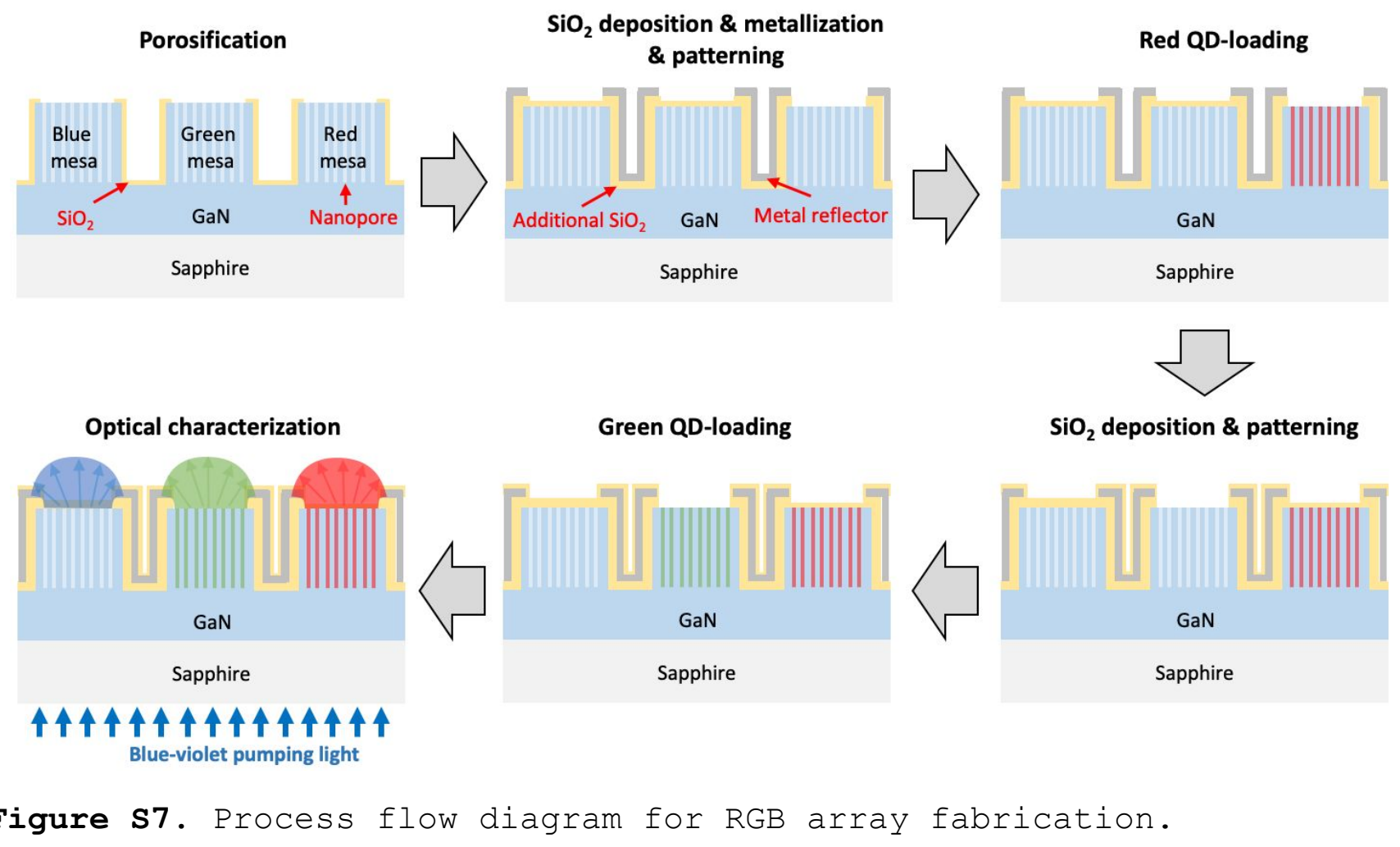

